\title{
Basic isoreticular metal-organic framework (IRMOF-3) porous nanomaterial as a suitable and green catalyst for selective unsymmetrical Hantzsch coupling reaction
}

\author{
Sadegh Rostamnia ${ }^{a *}$ and Hongchuan Xin ${ }^{b}$
}

A catalytic amount of the basic metal-organic framework (IRMOF-3) with organic substrates was found to be an efficient, selective and waste-free green approach for the unsymmetrical Hantzsch coupling reaction. The catalyst can be isolated from the reaction mixture and reused at least four times. Copyright $\odot 2014$ John Wiley \& Sons, Ltd.

Additional supporting information may be found in the online version of this article at the publisher's web-site.

Keywords: inorganic-organic hybrid material; metal-organic framework; IRMOF-3; unsymmetrical Hantzsch coupling reaction

\section{Introduction}

Metal-organic frameworks (MOFs) have been given much attention from both scientific and commercial aspects regarding their application to gas storage, gas separation and catalytic reaction. ${ }^{[1]}$ MOF-assisted organic synthesis using organicinorganic hybrid micro-channels has been utilized not only to accelerate a number of synthetic reactions, but also it is a green catalyst to increase reaction rate and yields. ${ }^{[2]}$ MOF-based structures demonstrate various advantages such as high surface area, adjustable pore size and the simplicity of processing, tunability and use of stable alternative materials. ${ }^{[2,3]}$ MOFs have been utilized as solid catalysts in reactions such as Friedel-Crafts alkylation and acylation, Sonogashira, alkene epoxidation, aldol, Suzuki, Biginelli, aza-Michael and Knoevenagel condensation. ${ }^{[4-6]}$ Recently, thermal and chemical stable $\mathrm{NH}_{2}$-MOFs have been reported and used as heterogeneous catalysts by Gascon, Xamena, Garcia, Corma and others. ${ }^{[4-6]}$ In these investigations, IRMOF-3 with non-coordinated amino groups demonstrates that the basicity of the aniline-like amino group is enhanced when incorporated inside the pores of MOF channels. ${ }^{[4-10]}$

Organic-inorganic hybrid porous IRMOF-3 would make materials hydrothermally stable and hydrophobic and thus provide improved catalytic properties during applications for organic small molecules. ${ }^{[8]}$ For these reasons and in our continuing interest in the synthesis and application of organic-inorganic hybrid nanoporous material in catalyst and coupling reaction fields, ${ }^{[11-17]}$ we report herein our results for the application of IRMOF-3 as a suitable, efficient and green catalyst for the multicomponent Hantzsch condensation of aldehyde, cyclic and acyclic $\beta$-diketenes with ammonia without any solvent, salts or additives, with excellent to good yields, for polyhydroquinolines of biological and medicinal ${ }^{[18]}$ interest (Fig. 1).

\section{Experimental}

\section{Chemicals and Apparatus}

All reagents were obtained from Merck (Germany) and Fluka (Switzerland) and were used without further purification. Melting points were measured on an Electrothermal 9100 apparatus. Progress of reactions was monitored by thin-layer chromatography (TLC). Mass spectra were recorded on a Finnigan-Matt 8430 mass spectrometer operating at an ionization potential of $70 \mathrm{eV}$. ${ }^{1} \mathrm{H}$ and ${ }^{13} \mathrm{C}$ NMR spectra were measured $\left(\mathrm{CDCl}_{3}\right)$ with a Bruker DRX-300 Avance spectrometer at 300 and $75 \mathrm{MHz}$, respectively.

\section{Preparation of IRMOF-3}

IRMOF-3 $\left(\mathrm{Zn}_{4} \mathrm{O}\left(\mathrm{H}_{2} \mathrm{~N}-\mathrm{TA}\right)_{3}\right)$ was synthesized according to the literature procedure, with slight modifications. ${ }^{[5,6]}$ In a typical catalyst preparation, $\mathrm{Zn}\left(\mathrm{NO}_{3}\right)_{2}(12.5 \mathrm{mmol})$ and $\mathrm{H}_{2} \mathrm{ATA}$ (4.1 mmol) were dissolved in dry DMF and stirred for $20 \mathrm{~min}$ at room temperature. The solution was transferred to a sealed Teflon-lined autoclave and kept at $100^{\circ} \mathrm{C}$ for $20 \mathrm{~h}$. The resulting brown solid was collected and washed with DMF and $\mathrm{CHCl}_{3}$, and finally dried under vacuum at $60^{\circ} \mathrm{C}$.

\footnotetext{
* Correspondence to: Sadegh Rostamnia, Organic and Nano Group (ONG), Department of Chemistry, Faculty of Science, University of Maragheh, PO Box, 55181-83111 Maragheh, Iran. E-mail: rostamnia@maragheh.ac.ir

a Organic and Nano Group (ONG), Department of Chemistry, Faculty of Science, University of Maragheh, Maragheh, 55181-83111, Iran

b Key Laboratory of Biofuels, Qingdao Institute of Bioenergy and Bioprocess Technology, Chinese Academy of Sciences, Qingdao, 266101, China
} 


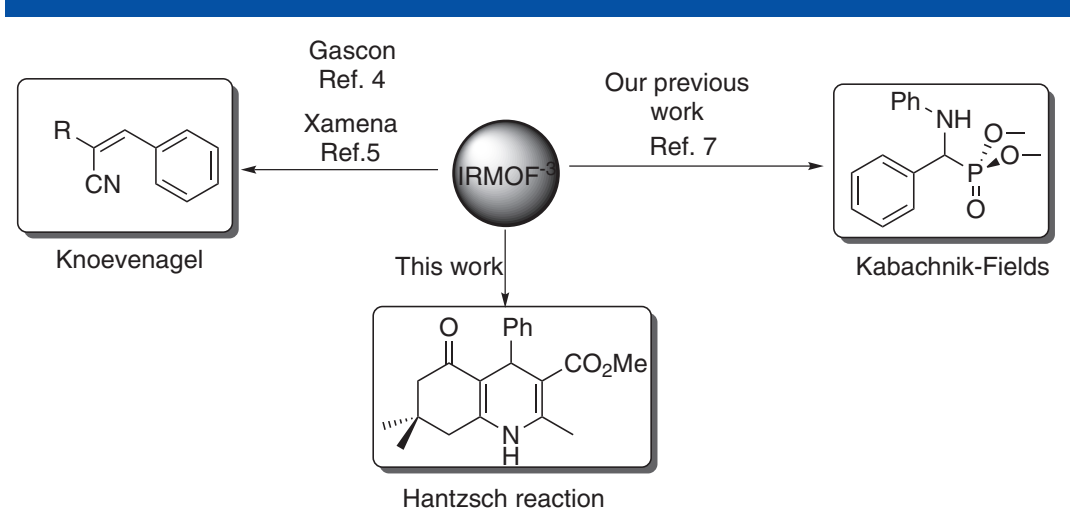

Figure 1. IRMOF-3 as porous solid-catalyst.

\section{General Procedure for the Synthesis of Polyhydroquinolines and Dihydropyridines}

A mixture of aldehyde ( $1 \mathrm{mmol}), \beta$-dicarbonyl compound ( 1 or $2 \mathrm{mmol}$ ) and $\mathrm{NH}_{4} \mathrm{OAC}(2.5 \mathrm{mmol})$ and dimedone $(1 \mathrm{mmol}$ or without) with a catalytic amount of IRMOF-3 $(0.04 \mathrm{~g}, 5 \mathrm{~mol} \%)$ under solvent free conditions was stirred at $60^{\circ} \mathrm{C}$. After complete disappearance of the starting material, as indicated by TLC, the resulting mixture was diluted with hot ethyl acetate $(10 \mathrm{ml})$ and filtered. The catalyst was completely recovered from the residue. All products were previously reported ${ }^{[16]}$ and were characterized by comparing IR, NMR and physical data with those reported (see supporting information).

\section{Catalyst Synthesis and Characterization}

The metal-organic framework IRMOF-3 was prepared by refluxing 2-aminoterephthalic acid $\left(\mathrm{H}_{2} \mathrm{ATA}\right)$ and zinc nitrate hexahydrate in dry DMF by a solvothermal method, according to a literature procedure. ${ }^{[5-8]}$ In a typical catalyst preparation, $\mathrm{Zn}\left(\mathrm{NO}_{3}\right)_{2}$ $(3.75 \mathrm{~g})$ and $\mathrm{H}_{2}$ ATA $(0.75 \mathrm{~g})$ were dissolved in DMF and the mixture was transferred to a Teflon-lined autoclave. The reaction mixture was kept for $1 \mathrm{~h}$ at $25^{\circ} \mathrm{C}$ and then at $100^{\circ} \mathrm{C}$ for $17 \mathrm{~h}$. The resulting brown solid was collected and washed three times with $\mathrm{CHCl}_{3}$, and the solid was finally dried under vacuum at $60^{\circ} \mathrm{C}$. The IRMOF-3 structure is made of $\mathrm{Zn}_{4} \mathrm{O}$ tetranuclear clusters connected by rigid $\mathrm{NH}_{2}$-benzenedicarboxylic linkers to generate a cubic framework (Fig. 2).<smiles>Nc1cc(C(=O)O)ccc1C(=O)O</smiles>
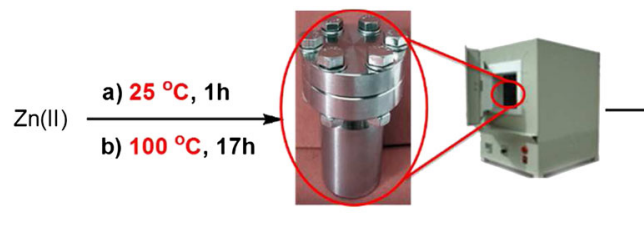

$\left(\mathrm{H}_{2} \mathrm{ATA}\right)$
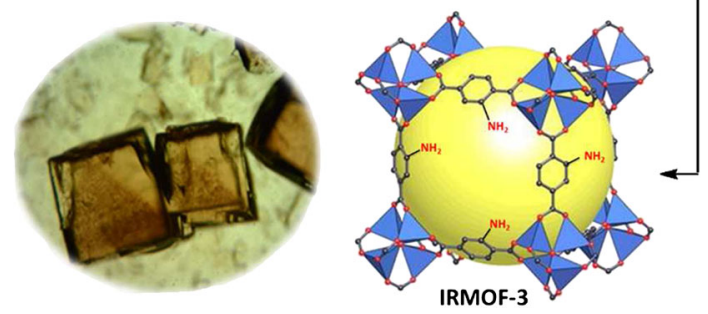

Figure 2. Preparation process of IRMOF-3.
The IRMOF-3 was then characterized using a variety of different techniques. The IR spectrum of IRMOF-3 shows two peaks in 3470 and $3359 \mathrm{~cm}^{-1}$ due to the existence of the amino groups of the $\mathrm{NH}_{2}-\mathrm{TA}$ ligand. ${ }^{[19]}$ The two sharp bands at 1575 and $1385 \mathrm{~cm}^{-1}$ correspond to asymmetric $\left(v_{\text {as }}(C-O)\right)$ and symmetric $\left(v_{s}\right.$ (C-O)) vibrations of carboxyl groups, respectively. ${ }^{[7]}$ The peaks centered at 16562, 1496 and $1421 \mathrm{~cm}^{-1}$ were ascribed to CC stretching vibration of the aromatic. The $1257 \mathrm{~cm}^{-1}$ frequency can be assigned to $\mathrm{C}-\mathrm{N}$ vibrations (Fig. 3c).

Diffraction peaks at around $2 \theta=6.8^{\circ}$ and $9.6^{\circ}$ are readily recognized from the $\mathrm{X}$-ray diffraction (XRD) pattern (Fig. 3d). The observed diffraction peaks agree with the IRMOF-3. ${ }^{[5-8]}$ The crystal structure of IRMOF-3 seems to change to amorphous through reusing four times. The nitrogen adsorption-desorption isotherms of the solvothermal synthesized IRMOF-3 are displayed in Fig. 3b. Optical microscope and SEM images (Figs $3 a$ and $4 a$ ), together with the XRD patterns show that IRMOF-3 was highly crystalline.

\section{Results and Discussion}

With regard to the chemical and biological interest of polyhydroquinolines, ${ }^{[18]}$ for synthesis of product 4 the efficacy of different catalysts was studied. As expected, adduct of dimedone and aldehydes treated with $\beta$-diketone, under the employed reaction conditions in the presence of the $\mathrm{NH}_{4} \mathrm{OAc}$ (Scheme 1), led to the products polyhydroquinolines $\mathbf{4 a}$ as an unsymmetrical Hantzsch coupling reaction and symmetrical dihydropyridines $\mathbf{5}$ and $6 .{ }^{[16]}$ For our study, dimedone, benzaldehyde, ammonium acetate and methyl acetylacetonate were chosen as the benchmark substrates in the model reaction.

In the model reaction, to obtain the desired product (4a) we tested the reaction under different conditions. IRMOF-3 was assessed for its catalytic activity in the unsymmetrical Hantzsch coupling reaction by studying the model reaction to produce of 4a as the principal product and symmetrical products $\mathbf{5}$ and $\mathbf{6}$ as by-product (Scheme 1). The model reaction was screened in polar and nonpolar solvents using different molar ratios of IRMOF-3. The results are summarized in Table 1. It should be emphasized that in this green waste-free combined catalytic system not a trace of byproduct $\mathbf{5}$ or $\mathbf{6}$ was observed in this reaction by TLC analysis. Due to the fact that the basic nature of the amine-decorated MOF (IRMOF-3) may play an important role in the synthesis of $\mathbf{4 a}$, in comparison with same non-amine isostructural MOF (MOF-5), the initial reaction was also carried out using 4 mol\% MOF-5 catalyst at $60^{\circ} \mathrm{C}$. IRMOF-3 was assessed for its catalytic activity in Hantzsch coupling by studying the model reaction for production of $\mathbf{4 a}$ as the principal product. When the model reaction was run using IRMOF-3, the product $4 \mathrm{a}$ was obtained in $91 \%$ yield in $3 \mathrm{~h}$.

In order to examine the scope of this process, and to demonstrate the diversity of IRMOF-3, the optimized conditions were applied to a series of substrates as shown in Table 2 . The catalytic activity of IRMOF-3 in the four-component synthesis of polyhydroquinolines, by condensation of dimedone, aldehydes, $\beta$-dicarbonyl compounds and ammonium acetate, was studied, in which adduct of dimedone and aldehyde was treated with 1,3-dicarbonyls in the presence of the ammonium acetate under 


\section{Basic metal-organic framework for unsymmetrical Hantzsch coupling}
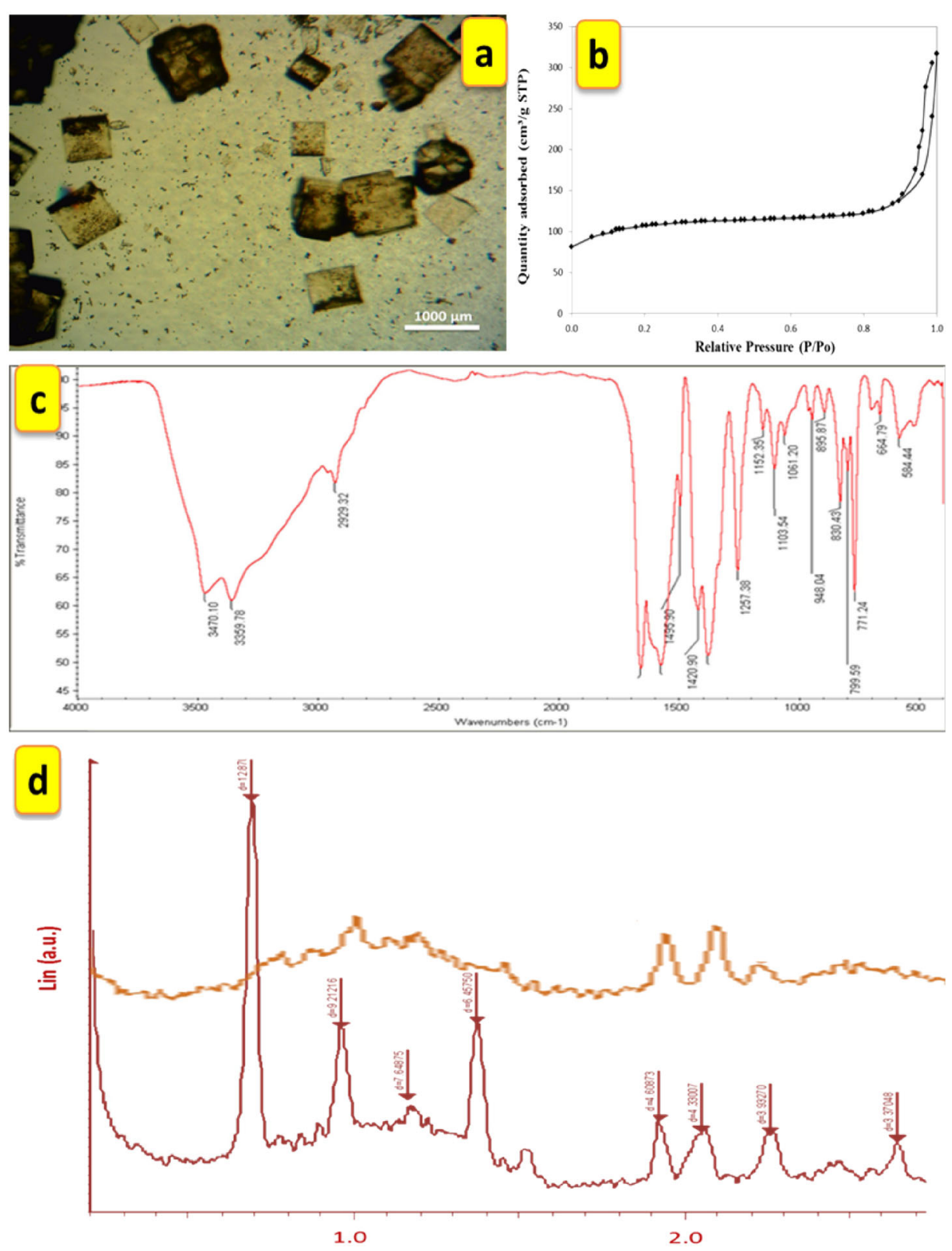

Figure 3. IRMOF-3 as nanoreactor: (a) optical photomicrograph; (b) nitrogen adsorption-desorption isotherms; (c) FT-IR pattern; (d) XRD patterns (brown, newly prepared; black, used four times for $\mathbf{4 a )}$ ).

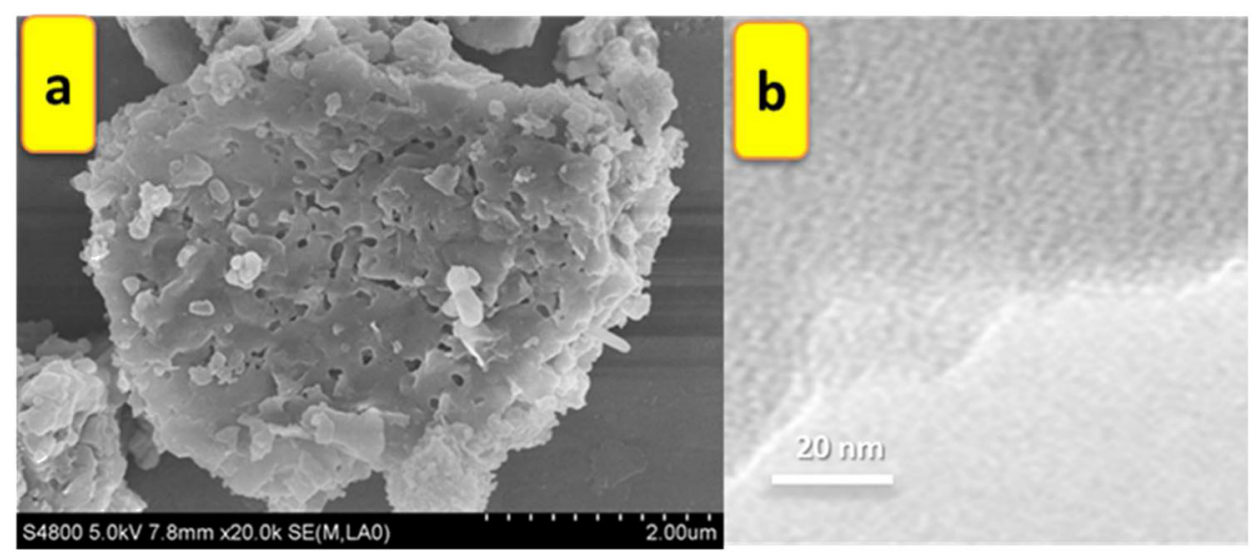

Figure 4. IRMOF-3 as catalyst: (a) SEM and (b) TEM images. 


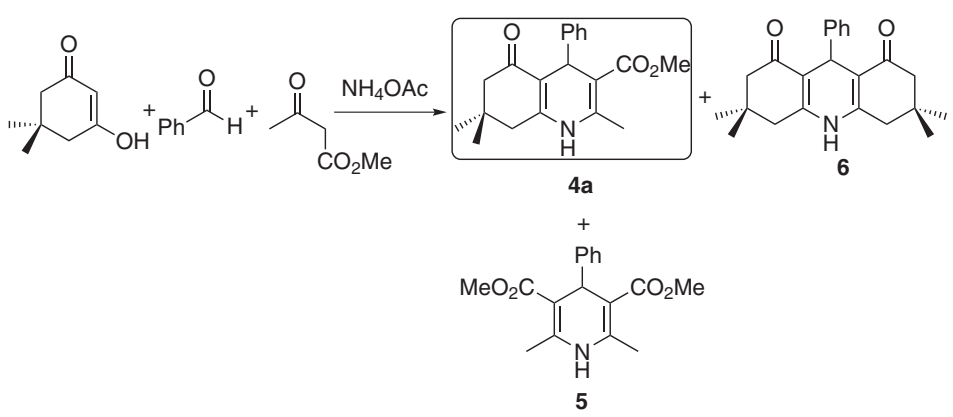

Scheme 1. Expected products from the reaction of dimedone, benzaldehyde, ammonium acetate and methyl acetylacetonate. produce polyhydroquinolines of pharmacologial and biological interest in an unsymmetrical Hantzch coupling reaction, in high yields under solvent-free conditions. This method has many advantages, such as short reaction time, small amount of catalyst needed and facile recycling, especially the lack of requirement for toxic organic solvents in the reaction, providing a green and effective method for synthesis of Hantzch polyhydroquinolines.

\begin{tabular}{|lllll|l|}
\hline Table 1. Different conditions in the four-component synthesis of \\
polyhydroquinoline 4a
\end{tabular}

the reaction conditions, which led to the polyhydroquinoline products 4.

The possibility of recycling and reusing the catalyst was then examined. In this case, after completion of the reactions (TLC), hot ethanol or $\mathrm{CH}_{2} \mathrm{Cl}_{2}$ was added and the catalyst was filtered and reused four times (Fig. 5).

\section{Conclusion}

We report a catalytic application of the porous nanomaterials of metal-organic frameworks (IRMOF-3) for multicomponent coupling of dimedone, aldehydes, $\beta$-diketone and ammonia to
Table 2. IRMOF-3-catalyzed synthesis of high-functionalized polyhydroquinolines 4

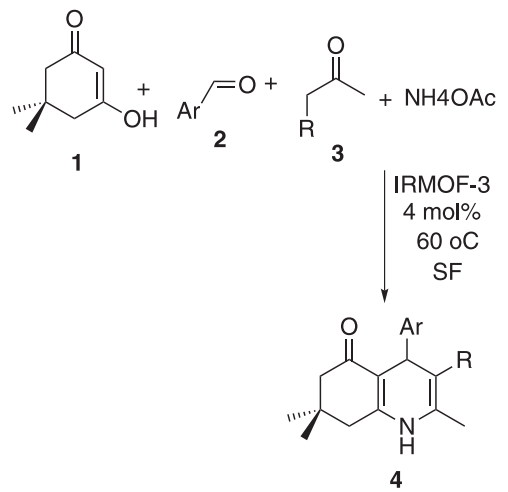

\begin{tabular}{lllccc|}
\hline Entry & \multicolumn{1}{c}{$\mathrm{Ar}$} & \multicolumn{1}{c}{$\mathrm{R}$} & Time $(\mathrm{h})$ & Yield (\% 4) & $\mathbf{4}$ \\
\hline 1 & $\mathrm{C}_{6} \mathrm{H}_{5}$ & $\mathrm{CO}_{2} \mathrm{Me}$ & 3 & 91 & $\mathbf{4 a}$ \\
2 & $2-\mathrm{ClC}_{6} \mathrm{H}_{4}$ & $\mathrm{CO}_{2} \mathrm{Me}$ & 3 & 93 & $\mathbf{4 b}$ \\
3 & $2-\mathrm{MeC}_{6} \mathrm{H}_{4}$ & $\mathrm{CO}_{2} \mathrm{Me}$ & 4 & 90 & $\mathbf{4 c}$ \\
4 & $4-\mathrm{ClC}_{6} \mathrm{H}_{4}$ & $\mathrm{CO}_{2} \mathrm{Me}$ & 3 & 93 & $\mathbf{4 d}$ \\
5 & $4-\mathrm{MeOC}_{6} \mathrm{H}_{4}$ & $\mathrm{CO}_{2} \mathrm{Me}$ & 5 & 85 & $\mathbf{4 e}$ \\
6 & $2-\mathrm{MeC}_{6} \mathrm{H}_{4}$ & $\mathrm{CO}_{2} \mathrm{Et}$ & 5 & 86 & $\mathbf{4 f}$ \\
7 & $2-\mathrm{ClC}_{6} \mathrm{H}_{4}$ & $\mathrm{CO}_{2} \mathrm{Et}$ & 3 & 91 & $\mathbf{4 g}$ \\
8 & $4-\mathrm{Cl}_{6} \mathrm{H}_{4}$ & $\mathrm{CO}_{2} \mathrm{Et}$ & 3 & 93 & $\mathbf{4 h}$ \\
9 & $4-\mathrm{NO}_{2} \mathrm{C}_{6} \mathrm{H}_{4}$ & $\mathrm{CO}_{2} \mathrm{Et}$ & 2.5 & 94 & $\mathbf{4 i}$ \\
10 & $4-\mathrm{MeOC}_{6} \mathrm{H}_{4}$ & $\mathrm{CO}_{2} \mathrm{Et}$ & 4 & 87 & $\mathbf{4 j}$ \\
& & & & & \\
\hline
\end{tabular}

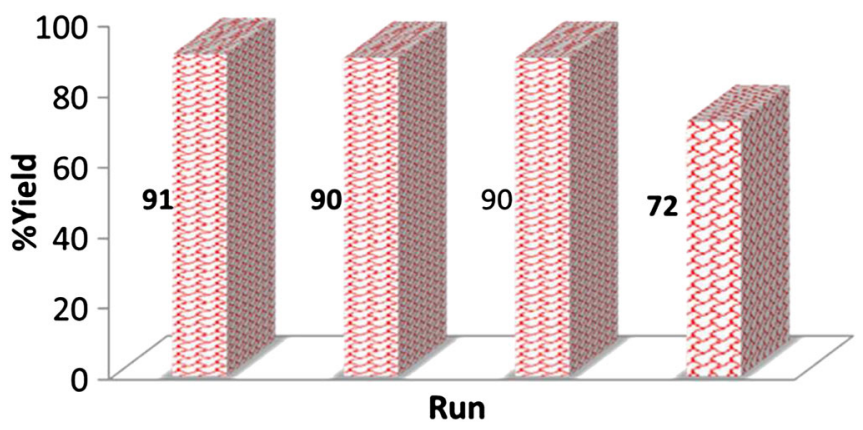

Figure 5. Recyclability of IRMOF-3 in the synthesis of $\mathbf{4 a}$. 


\section{References}

[1] H. Li, M. Eddaoudi, M. O'Keeffe, O. M. Yaghi, Nature 1999, 402, 276-279.

[2] a) J. L. C. Rowsell, O. M. Yaghi, Micropor. Mesopor. Mater. 2004, 73, 3-14; b) M. Y. Masoomi, A. Morsali, Coord. Chem. Rev. 2012, 256, 2921-2943; c) A. Morsali, M. Y. Masoomi, Coord. Chem. Rev. 2009, 253, 1882-1905; d) M. Y. Masoomi, A. Morsali, RSC Adv. 2013, 3, 19191-19218; e) M. Y. Masoomi, G. Mahmoudi, A. Morsali, J. Coord. Chem. 2010, 63, 1186-1193.

[3] Z. Q. Li, L. G. Qiu, T. Xu, Y. Wu, W. Wang, Z. Y. Wu, X. Jiang, Mater. Lett. 2009, 63, 78-80.

[4] J. Gascon, U. Aktay, M. D. Hernandez-Alonso, G. P. M. van Klink, F. Kapteijn, J. Catal. 2009, 261, 75-87.

[5] F. X. L. Xamena, A. Abad, A. Corma, H. Garcia, J. Catal. 2007, 250, 294-298.

[6] F. X. L. Xamena, F. G. Cirujano, A. Corma, Micropor. Mesopor. Mater. 2012, 157, 112-117.

[7] S. Rostamnia, H. Xin, N. Nouruzi, Micropor. Mesopor. Mater. 2013, 179, 99-103.

[8] P. Serra-Crespo, E. V. Ramos-Fernandez, J. Gascon, F. Kapteijn, Chem. Mater. 2011, 23, 2565-2572.

[9] M. De Lange, J. Gutierrez-Sevillano, S. Hamad, T. Vlugt, S. Calero, J. Gascon, F. Kapteijn, J. Phys. Chem. C 2013, 117, 7613-7622.
[10] P. Serra-Crespo, E. Gobechiya, E. V. Ramos-Fernandez, J. Juan-Alcañiz, A. Martinez-Joaristi, E. Stavitski, C. Kirschhock, J. Martens, F. Kapteijn, J. Gascon, Langmuir 2012, 285, 12916-12922.

[11] S. Rostamnia, K. Lamei, M. Mohammadquli, M. Sheykhan, A. Heydari, Tetrahedron Lett. 2012, 53, 5257-5260.

[12] S. Rostamnia, K. Lamei, Synthesis 2011, 3080-3082.

[13] S. Rostamnia, A. Zabardasti, J. Fluorine Chem. 2012, 144, 69-72.

[14] S. Rostamnia, E. Doustkhah, A. Nuri, J. Fluorine Chem. 2013, 153, 1-6.

[15] S. Rostamnia, A. Nuri, H. Xin, A. Pourjavadi, S. H. Hosseini, Tetrahedron Lett. 2013, 54, 3344-3347.

[16] S. Rostamnia, H. Xin, L. Xiao, K. Lamei, J. Mol. Catal A: Chem. 2013, 374-375, 85-93.

[17] S. Rostamnia, H. Xin, Appl. Organometal. Chem. 2013, 27, 348-352.

[18] I. Yalcin, I. Oren, E. Sener, A. Akin, N. Ucarturk, Eur. J. Med. Chem. 1992, 27, 395.

[19] H. Xin, J. Liu, F. Fan, Z. Feng, G. Jia, Q. Yang, C. Li, Micropor. Mesopor. Mater. 2008, 113, 231-239.

\section{Supporting Information}

Additional supporting information may be found in the online version of this article at the publisher's web-site. 